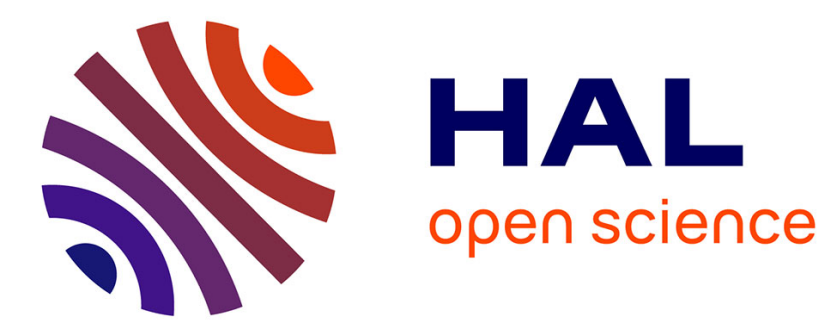

\title{
An iterative approach to non-overdetermined inverse scattering at fixed energy \\ Roman Novikov
}

\section{To cite this version:}

Roman Novikov. An iterative approach to non-overdetermined inverse scattering at fixed energy. Sbornik: Mathematics, 2015, 206 (1), pp.120-134. hal-00835735

\section{HAL Id: hal-00835735 https://hal.science/hal-00835735}

Submitted on 19 Jun 2013

HAL is a multi-disciplinary open access archive for the deposit and dissemination of scientific research documents, whether they are published or not. The documents may come from teaching and research institutions in France or abroad, or from public or private research centers.
L'archive ouverte pluridisciplinaire HAL, est destinée au dépôt et à la diffusion de documents scientifiques de niveau recherche, publiés ou non, émanant des établissements d'enseignement et de recherche français ou étrangers, des laboratoires publics ou privés. 


\section{An iterative approach to non-overdetermined inverse scattering at fixed energy}

\section{R.G. Novikov}

CNRS (UMR 7641), Centre de Mathématiques Appliquées, Ecole Polytechnique, 91128 Palaiseau, France, and IEPT RAS, 117997 Moscow, Russia e-mail: novikov@cmap.polytechnique.fr

Abstract. We propose an iterative approximate reconstruction algorithm for nonoverdetermined inverse scattering at fixed energy $E$ with incomplete data in dimension $d \geq 2$. In particular, we obtain rapidly converging approximate reconstructions for this inverse scattering for $E \rightarrow+\infty$.

\section{Introduction}

We consider the Schrödinger equation

$$
H \psi=E \psi, \quad H=-\Delta+v(x), \quad x \in \mathbb{R}^{d}, d \geq 2, E>0
$$

where

$$
v \in L_{\sigma}^{\infty}\left(\mathbb{R}^{d}\right) \text { for some } \sigma>d
$$

where

$$
\begin{aligned}
& L_{\sigma}^{\infty}\left(\mathbb{R}^{d}\right)=\left\{u \in L^{\infty}\left(\mathbb{R}^{d}\right):\|u\|_{\sigma}<+\infty\right\}, \\
& \|u\|_{\sigma}=\text { ess } \sup _{x \in \mathbb{R}^{d}}\left(1+|x|^{2}\right)^{\sigma / 2}|u(x)|, \sigma \geq 0 .
\end{aligned}
$$

For equation (1.1) we consider the classical scattering eigenfunctions $\psi^{+}$specified by the following asymtotics as $|x| \rightarrow \infty$ :

$$
\begin{aligned}
& \psi^{+}(x, k)=e^{i k x}+c(d,|k|) \frac{e^{i|k||x|}}{|x|^{(d-1) / 2}} f\left(k,|k| \frac{x}{|x|}\right)+o\left(\frac{1}{|x|^{(d-1) / 2}}\right), \\
& x \in \mathbb{R}^{d}, k \in \mathbb{R}^{d}, k^{2}=E, c(d,|k|)=-\pi i(-2 \pi i)^{(d-1) / 2}|k|^{(d-3) / 2},
\end{aligned}
$$

where a priori unknown function $f=f(k, l), k, l \in \mathbb{R}^{d}, k^{2}=l^{2}=E$, arising in (1.4) is the classical scattering amplitude for (1.1).

Given potential $v$, to determine $\psi^{+}$and $f$ one can use, in particular, the LippmannSchwinger integral equation

$$
\begin{aligned}
& \psi^{+}(x, k)=e^{i k x}+\int_{\mathbb{R}^{d}} G^{+}(x-y, k) v(y) \psi^{+}(y, k) d y, \\
& G^{+}(x, k)=-(2 \pi)^{-d} \int_{\mathbb{R}^{d}} \frac{e^{i \xi x} d \xi}{\xi^{2}-k^{2}-i 0},
\end{aligned}
$$


and the formula

$$
f(k, l)=(2 \pi)^{-d} \int_{\mathbb{R}^{d}} e^{-i l y} v(y) \psi^{+}(y, k) d y,
$$

where $x, k, l \in \mathbb{R}^{d}, k^{2}=l^{2}=E>0$; see, for example, [BS], [F2].

The scattering amplitude $f$ at fixed energy $E>0$ is defined on

$$
\mathcal{M}_{E}=\left\{k \in \mathbb{R}^{d}, l \in \mathbb{R}^{d}: \quad k^{2}=l^{2}=E\right\}, \quad E>0
$$

Following [N8], in addition to $f$ on $\mathcal{M}_{E}$ we consider also $\left.f\right|_{\Gamma_{E}}$ and $\left.f\right|_{\Gamma_{E}^{\tau}}$, where

$$
\begin{gathered}
\Gamma_{E}=\left\{k=k_{E}(p), l=l_{E}(p): p \in \mathcal{B}_{2 \sqrt{E}}\right\}, \\
\Gamma_{E}^{\tau}=\left\{k=k_{E}(p), l=l_{E}(p): p \in \mathcal{B}_{2 \tau} \sqrt{E}\right\}, \\
k_{E}(p)=\frac{p}{2}+\eta_{E}(p), l_{E}(p)=-\frac{p}{2}+\eta_{E}(p), \\
\mathcal{B}_{r}=\left\{p \in \mathbb{R}^{d}:|p| \leq r\right\}, \quad r>0,
\end{gathered}
$$

where $E>0,0<\tau \leq 1, d \geq 2$, and $\eta_{E}$ is a piecewise continuous vector-function on $\mathcal{B}_{2 \sqrt{E}}$ such that

$$
\eta_{E}(p) p=0, \quad \frac{p^{2}}{4}+\left(\eta_{E}(p)\right)^{2}=E, \quad p \in \mathcal{B}_{2 \sqrt{E}}
$$

One can see that

$$
\Gamma_{E}^{\tau} \subseteq \Gamma_{E} \subset \mathcal{M}_{E}, E>0,0<\tau \leq 1, d \geq 2 .
$$

In this work we continue studies on the following inverse scattering problems for equation (1.1) under assumptions (1.2):

Problem 1.1. Given scattering amplitude $f$ on $\mathcal{M}_{E}$ at fixed $E>0$, find potential $v$ on $\mathbb{R}^{d}$ (at least approximately).

Problem 1.2. Given scattering amplitude $f$ on $\Gamma_{E}^{\tau}$ at fixed $E$ and $\tau$, where $E>0$, $0<\tau \leq 1$, find potential $v$ on $\mathbb{R}^{d}$ (at least approximately).

In addition, one can see that

$$
\begin{aligned}
& \operatorname{dim} \mathcal{M}_{E}=2 d-2, \operatorname{dim} \Gamma_{E}^{\tau}=\operatorname{dim} \Gamma_{E}=\operatorname{dim} \mathbb{R}^{d}=d \text { for } d \geq 2, \\
& \operatorname{dim} \mathcal{M}_{E}>d \text { for } d \geq 3
\end{aligned}
$$

where $E>0,0<\tau \leq 1$. Therefore, Problem 1.1 is overdetermined for $d \geq 3$, whereas Problem 1.2 is non-overdetermined.

Problem 1.1 has a long history and there are many important results on this problem, see $[\mathrm{ABR}],[\mathrm{B}],[\mathrm{BAR}],[\mathrm{ChS}],[\mathrm{E}],[\mathrm{F} 1],[\mathrm{GHN}],[\mathrm{G}],[\mathrm{HH}],[\mathrm{I}],[\mathrm{IN}],[\mathrm{N} 1]-[\mathrm{N} 5],[\mathrm{R}],[\mathrm{S} 1]$, $[\mathrm{VW}],[\mathrm{W}],[\mathrm{WY}]$ and references therein. Note also that for spherical potentials $v$ Problem 1.2 for $\tau=1$ is reduced to Problem 1.1. However, to our knowledge, explicit considerations of Problem 1.2 were started only recently in [N8]. In addition, concerning known results for 
some other non-overdetermined multi-dimensional coefficient inverse problems, see [BK], [DKN], [ER1], [HN], [K], [M], [N6], [NS], [S2] and references therein.

Note also that Problems 1.1, 1.2 can be considered as examples of ill-posed problems; see $[\mathrm{BK}],[\mathrm{LRS}]$ for an introduction to this theory.

In the present work we consider Problems 1.1, 1.2 assuming that

$v$ is a perturbation of some known background $v_{0}$ satisfying (1.2), where $v-v_{0}$ is sufficiently regular on $\mathbb{R}^{d}$ and $\operatorname{supp}\left(v-v_{0}\right) \subset D$,

where $D$ is an open bounded domain (which is fixed a priori).

In particular, for Problem 1.2, under assumptions (1.13), we iteratively construct stable approximations $u_{j}(x, E)$ to the unknown $v(x), x \in D$, where $u_{1}$ is a linear reconstruction in the Born approximation and $u_{j}, j \geq 2$, are non-linear approximate reconstructions from $f$ on $\Gamma_{E}^{\tau}$; see Subsections 3.2, 3.3. Our construction is based on direct scattering results (summarized in Section 2) and on standard Fourier analysis. In addition, our non-linear approximate reconstructions $u_{j}$ are efficient in the sense that

$$
\left\|u_{j}(\cdot, E)-v\right\|_{L^{\infty}(D)}=\varepsilon_{j}(E)
$$

rapidly decay as $E \rightarrow+\infty$, for sufficiently regular $v-w$ and sufficiently large $j$. In particular,

$$
\begin{aligned}
& \varepsilon_{j}(E)=O\left(E^{-\alpha_{j}}\right), \quad \alpha_{j}=\left(1-\left(\frac{n-d}{n}\right)^{j}\right) \frac{n-d}{2 d}, \\
& \text { as } E \rightarrow+\infty, \quad j \geq 1,
\end{aligned}
$$

if $v-v_{0}$ is $n$-times smooth in $L^{1}\left(\mathbb{R}^{d}\right), n>d$; see Theorem 3.1 of Subsection 3.4. Note that $u_{j}$ and $\varepsilon_{j}$ depend also on fixed $\tau$ of Problem 1.2.

In addition, in Subsection 3.5 we explain that the construction of $u_{j}$ for each $j \in \mathbb{N}$ can be reduced to a finite number of explicit formulas; see Subsection 3.5 for details.

It is also important to note that $f$ on $\Gamma_{E}^{\delta(E)}$ only is used in our iterative approximate reconstruction for Problem 1.2 at high energies $E$, where

$$
\left.\left.\delta(E)=\tau E^{-(d-1) /(2 d)}, \quad \tau \in\right] 0,1\right]
$$

In addition, $\delta(E) \rightarrow 0$ as $E \rightarrow+\infty$. Therefore, $\Gamma_{E}^{\delta(E)}$ is a very small part of $\Gamma_{E}^{\tau_{1}}$ for any fixed $\left.\left.\tau_{1} \in\right] 0,1\right]$ for sufficiently large $E$. Therefore, our iterative approximate reconstruction can be viewed as a reconstruction result for Problem 1.2 with incomplete data.

Actually, the iterative approximate reconstruction of the present work complements related stability results of [N8].

In addition, the iterative reconstruction of the present work was also influenced by the iterative reconstruction of [N7] for quite different inverse scattering problem.

Let us consider also

$$
\left.\left.\mathcal{M}_{E}^{\tau}=\left\{(k, l) \in \mathcal{M}_{E}: k-l \in \mathcal{B}_{2 \tau \sqrt{E}}\right\}, E>0, \tau \in\right] 0,1\right]
$$


Note that

$$
\begin{aligned}
& \left.\left.\Gamma_{E}^{\tau} \subset \mathcal{M}_{E}^{\tau} \text { for } \tau \in\right] 0,1\right] \\
& \left.\mathcal{M}_{E}^{\tau} \subset \mathcal{M}_{E} \text { for } \tau \in\right] 0,1\left[, \mathcal{M}_{E}^{\tau}=\mathcal{M}_{E} \text { for } \tau=1,\right. \\
& \left.\left.\operatorname{dim} \mathcal{M}_{E}^{\tau}=\operatorname{dim} \mathcal{M}_{E}=2 d-2 \text { for } \tau \in\right] 0,1\right] .
\end{aligned}
$$

To our knowledge, no analog of the non-linear approximate reconstructions $u_{j}, j \geq 2$, was given in the iterature even for

Problem 1.1 with $\mathcal{M}_{E}$ replaced by $\mathcal{M}_{E}^{\tau}$ for some fixed $\left.\tau \in\right] 0,1[$, i.e. for the problem with much richer data than Problem 1.2 (and, especially, than Problem 1.2 with $f$ given on $\Gamma_{E}^{\delta(E)}$ only, where $\delta(E)$ is defined by $\left.(1.16)\right)$.

On the other hand, for the case of Problem 1.1 with complete data, under assumptions (1.13), where $v-v_{0}$ is $n$-times smooth in $L^{1}\left(\mathbb{R}^{d}\right), n>d$, and $v_{0} \equiv 0$, the non-linear approximate reconstructions $u_{j}, j \geq 2$, of the present work are less precise than the approximate reconstructions of [N4], [N5] with the error term estimated as $O\left(E^{-s}\right)$ in the uniform norm as $E \rightarrow+\infty$, where $s=(n-d) / 2$ for $d=2, s=(n-d-\delta) / 2$ for any fixed arbitrary small $\delta>0$ for $d=3$. Indeed,

$$
\alpha_{j}<\frac{n-d}{2 d}<s
$$

where $\alpha_{j}$ are the numbers of (1.15), $s$ is the number of [N4], [N5].

However, for the problem of approximate but stable finding $v$ on $\mathbb{R}^{d}$ from $f$ on $\Gamma_{E}^{\delta(E)}$ (or even on $\mathcal{M}_{E}^{\delta(E)}$ ) only, where $\delta(E)$ is defined by (1.16), our approximate reconstructions $u_{j}$ for sufficiently large $j$ are rather optimal with respect to their precision (1.14), (1.15) even in the framework of standards of the Born approximation.

Indeed, in the Born approximation (linear approximation near $v_{0} \equiv 0$ ) the problem of finding $v$ on $\mathbb{R}^{d}$ from $f$ on $\mathcal{M}_{E}^{\delta}$, where $\left.E>0, \delta \in\right] 0,1[, d \geq 2$, is reduced to finding $v$ on $\mathbb{R}^{d}$ from its Fourier transform $\hat{v}$ on $\mathcal{B}_{2 \delta \sqrt{E}}$, where

$$
\hat{v}(p)=(2 \pi)^{-d} \int_{\mathbb{R}^{d}} e^{i p x} v(x) d x, \quad p \in \mathbb{R}^{d} .
$$

This linearized inverse scattering problem can be solved by the formula

$$
\begin{aligned}
& v(x)=v_{a p p r}^{\text {lin }}(x, E, \delta)+v_{\text {err }}^{\text {lin }}(x, E, \delta), \quad x \in \mathbb{R}^{d}, \\
& v_{\text {appr }}^{\text {lin }}(x, E, \delta)=\int_{\mathcal{B}_{2 \delta \sqrt{E}}} e^{-i p x} \hat{v}(p) d p, \\
& v_{\text {err }}^{\text {lin }}(x, E, \delta)=\int_{\mathbb{R}^{d} \backslash \mathcal{B}_{2 \delta \sqrt{E}}} e^{-i p x} \hat{v}(p) d p .
\end{aligned}
$$

In addition, we have that:

$$
\begin{aligned}
& \varepsilon(E, \delta) \stackrel{\text { def }}{=}\left\|v_{\text {err }}^{\text {lin }}(x, E, \delta)\right\|_{L^{\infty}\left(\mathbb{R}^{d}\right)}= \\
& O\left((\delta \sqrt{E})^{-(n-d)}\right) \text { if } \delta \sqrt{E} \rightarrow+\infty
\end{aligned}
$$


under the assuption that $v$ is $n$-times smooth in $L^{1}\left(\mathbb{R}^{d}\right), n>d$. In addition,

$$
\varepsilon(E, \delta(E))=O\left((\delta(E) \sqrt{E})^{-(n-d)}\right)=O\left(E^{-\alpha}\right), \quad \alpha=\frac{n-d}{2 d}, E \rightarrow+\infty
$$

for $\delta(E)$ given by $(1.16)$.

Finally, one can see that $\alpha_{j} \rightarrow \alpha$ for $j \rightarrow+\infty$, where $\alpha_{j}$ are the numbers of (1.15) and $\alpha$ is the number of the Born approximation estimates (1.22), (1.23).

\section{Preliminaries of direct scattering}

In this section we summarize some results related with the Lippmann- Schwinger integral equation (1.5) for the scattering eigenfunctions $\psi^{+}$and with formula (1.6) for the scattering amplitude $f$.

It is convenient to write the Lippmann-Schwinger integral equation (1.5) as

$$
(I-A(k)) \varphi(\cdot, k)=e(\cdot, k) \text {, }
$$

where

$$
\begin{aligned}
& \varphi(x, k)=\Lambda^{-\sigma / 2} \psi^{+}(x, k), \quad e(x, k)=\Lambda^{-\sigma / 2} e^{i k x}, \\
& A(k)=\Lambda^{-\sigma / 2} G^{+}(k) \Lambda^{-\sigma / 2}\left(\Lambda^{\sigma} v\right), k \in \mathbb{R}^{d} \backslash\{0\}, x \in \mathbb{R}^{d},
\end{aligned}
$$

where $I$ is the identity operator, $\Lambda$ denotes the multiplication operator by the functions $\left(1+|x|^{2}\right)^{1 / 2}, G^{+}$denotes the integral operator with the Schwartz kernel $G^{+}(x-y, k)$ of (1.5), $v$ is the multiplication operator by the function $v(x), \sigma$ is the number of (1.2). In addition, we recall that the following estimate holds:

$$
\begin{aligned}
& \left\|\Lambda^{-s} G^{+}(k) \Lambda^{-s}\right\|_{L^{2}\left(\mathbb{R}^{2}\right) \rightarrow L^{2}\left(\mathbb{R}^{2}\right)} \leq a_{0}(d, s)|k|^{-1}, \\
& k \in \mathbb{R}^{d},|k| \geq 1, \text { for } s>1 / 2,
\end{aligned}
$$

see $[\mathrm{E}],[\mathrm{J}]$ and references therein.

Using (2.3) one can see that

$$
\|A(k)\|_{L^{2}\left(\mathbb{R}^{d}\right) \rightarrow L^{2}\left(\mathbb{R}^{d}\right)} \leq a_{0}(d, \sigma / 2)\|v\|_{\sigma}|k|^{-1}, \quad k \in \mathbb{R}^{d},|k| \geq 1,
$$

where $\|\cdot\|_{\sigma}$ is defined in (1.3).

As a corollary of $(2.1),(2.2),(2.4)$, we have that

$$
\begin{aligned}
& \left\|\varphi(\cdot, k)-\sum_{j=0}^{m}(A(k))^{j} e(\cdot, k)\right\|_{L^{2}\left(\mathbb{R}^{d}\right)} \leq 2\left(\frac{a_{0}(d, \sigma / 2)\|v\|_{\sigma}}{|k|}\right)^{m+1} c_{1}(d) \\
& \text { for } k \in \mathbb{R}^{d},|k| \geq \rho_{1}\left(d, \sigma,\|v\|_{\sigma}\right), \quad m \in \mathbb{N} \cup 0
\end{aligned}
$$

where

$$
\begin{aligned}
& c_{1}(d, \sigma)=\|e(\cdot, k)\|_{L^{2}\left(\mathbb{R}^{d}\right)}=\left(\int_{\mathbb{R}^{d}} \frac{d x}{\left(1+|x|^{2}\right)^{\sigma / 2}}\right)^{1 / 2}, \\
& \rho_{1}(d, \sigma, N)=\max \left(2 a_{0}(d, \sigma / 2) N, 1\right) .
\end{aligned}
$$


Actually, formula (2.5) is a well-known method for solving the Lippmann-Schwinger integral equation (1.5) for sufficiently high energies.

Let now $\varphi_{i}, A_{i}$ denote $\varphi, A$ for $v=v_{i}$ satisfying (1.2), where $i=1,2$. Using the identity

$$
\begin{aligned}
& \left(I-A_{2}(k)\right)^{-1}-\left(I-A_{1}(k)\right)^{-1}= \\
& \left(I-A_{2}(k)\right)^{-1}\left(A_{2}(k)-A_{1}(k)\right)\left(I-A_{1}(k)\right)^{-1},
\end{aligned}
$$

one can see that

$$
\begin{aligned}
& \varphi_{2}(\cdot, k)-\varphi_{1}(\cdot, k)= \\
& \left(I-A_{2}(k)\right)^{-1}\left(A_{2}(k)-A_{1}(k)\right)\left(I-A_{1}(k)\right)^{-1} e(\cdot, k) .
\end{aligned}
$$

Using (2.2), (2.3), (2.4), (2.8), we obtain that

$$
\begin{aligned}
& \left\|\varphi_{2}(\cdot, k)-\varphi_{1}(\cdot, k)\right\|_{L^{2}\left(\mathbb{R}^{d}\right)} \leq 4 a_{0}(d, \sigma / 2)\left\|v_{2}-v_{1}\right\|_{\sigma} c_{1}(d, \sigma)|k|^{-1} \\
& \text { for } k \in \mathbb{R}^{d},|k| \geq \rho_{1}(d, \sigma, N),
\end{aligned}
$$

where $c_{1}, \rho_{1}$ are defined in (2.6), $\left\|v_{i}\right\|_{\sigma} \leq N$ for $i=1,2$.

Due to (1.6), we have also that

$$
\hat{v}(k-l)=f(k, l)-(2 \pi)^{-d} \int_{\mathbb{R}^{d}} e^{-i l x} v(x)\left(\psi^{+}(x, k)-e^{i k x}\right) d x, \quad(k, l) \in \mathcal{M}_{E}
$$

where $\hat{v}$ is defined by (1.20).

In particular, as a corollary of (2.10) and (2.5) for $m=0$, we have that

$$
\begin{aligned}
& |f(k, l)-\hat{v}(k-l)| \leq 2(2 \pi)^{-d} a_{0}(d, \sigma / 2)\left(c_{1}(d, \sigma)\|v\|_{\sigma}\right)^{2} E^{-1 / 2} \\
& (k, l) \in \mathcal{M}_{E}, \quad E^{1 / 2} \geq \rho_{1}\left(d, \sigma,\|v\|_{\sigma}\right)
\end{aligned}
$$

where $c_{1}, \rho_{1}$ are defined in (2.6).

Our iterative reconstruction algorithm for Problem 1.2 is based on formulas (2.5), (2.9), (2.10), (2.11) and is presented in the next section.

\section{Iterative approximate reconstruction for Problem $\mathbf{1 . 2}$}

3.1. Assumptions and notations. We consider

$$
\begin{aligned}
& W^{n, 1}\left(\mathbb{R}^{d}\right)=\left\{u: \partial^{J} u \in L^{1}\left(\mathbb{R}^{d}\right),|J| \leq n\right\} \\
& \|u\|_{n, 1}=\max _{|J| \leq n}\left\|\partial^{J} u\right\|_{L^{1}\left(\mathbb{R}^{d}\right)}, n \in \mathbb{N} \cup 0,
\end{aligned}
$$

where

$$
J \in(\mathbb{N} \cup 0)^{d},|J|=\sum_{i=1}^{d} J_{i}, \partial^{J} u(x)=\frac{\partial^{|J|} u(x)}{\partial x_{1}^{J_{1}} \ldots \partial x_{d}^{J_{d}}}
$$


An iterative approach to non-overdetermined inverse scattering at fixed energy

We assume that $v$ satisfies (1.13), where the assumption that $v-v_{0}$ is sufficiently regular is specified as

$$
v-v_{0} \in W^{n, 1}\left(\mathbb{R}^{d}\right) \text { for some } n>d \text {. }
$$

We set

$$
w=v-v_{0}
$$

and consider the decompositions

$$
\begin{aligned}
& v(x)=v^{+}(x, \kappa)+v^{-}(x, \kappa), \\
& v_{0}(x)=v_{0}^{+}(x, \kappa)+v_{0}^{-}(x, \kappa), \\
& w(x)=w^{+}(x, \kappa)+w^{-}(x, \kappa),
\end{aligned}
$$

where $x \in D, \kappa>0$,

$$
\begin{aligned}
& u^{+}(x, \kappa)=\int_{p \in \mathbb{R}^{d},|p| \leq \kappa} e^{-i p x} \hat{u}(p) d p, \\
& u^{-}(x, \kappa)=\int_{p \in \mathbb{R}^{d},|p|>\kappa} e^{-i p x} \hat{u}(p) d p, \\
& \hat{u}(p)=(2 \pi)^{-d} \int_{\mathbb{R}^{d}} e^{i p x} u(x) d x
\end{aligned}
$$

for $u=v, v_{0}, w$.

Due to (3.3)-(3.5), we have that

$$
\begin{aligned}
& v(x)=v^{+}(x, \kappa)+v_{0}^{-}(x, \kappa)+w^{-}(x, \kappa), \\
& v^{+}(x, \kappa)=v_{0}^{+}(x, \kappa)+w^{+}(x, \kappa),
\end{aligned}
$$

where $x \in D, \kappa>0$.

3.2. Reconstruction in the Born approximation. We define

$$
\begin{aligned}
& v_{1}\left(x, E, \tau_{1}\right) \stackrel{\text { def }}{=} v_{1}^{+}\left(x, E, \tau_{1}\right)+v_{0}^{-}\left(x, 2 \tau_{1} \sqrt{E}\right), \\
& v_{1}^{+}\left(x, E, \tau_{1}\right) \stackrel{\text { def }}{=} \int_{p \in \mathbb{R}^{d},|p| \leq 2 \tau_{1} \sqrt{E}} e^{-i p x} f\left(k_{E}(p), l_{E}(p)\right) d p, \\
& x \in D, \quad 0<\tau_{1} \leq \tau, \quad E>0,
\end{aligned}
$$

where $v_{0}^{-}$is the function of (3.4), (3.6), $f$ is the scattering amplitude for $v$, and $k_{E}, l_{E}$ are defined in (1.8). Actually, $v_{1}$ of (3.8a) is a reconstruction in the Born approximation for Problem 1.2.

Lemma 3.1. Let $v$ satisfy (1.13), (3.2) and $\|v\|_{\sigma} \leq M_{1},\left\|v-v_{0}\right\|_{n, 1} \leq M_{2}$. Then:

$$
\begin{aligned}
& \left|v_{1}\left(x, E, \tau_{1}\right)-v(x)\right| \leq c_{2}(d, \sigma) M_{1}^{2} \frac{\left(2 \tau_{1} \sqrt{E}\right)^{d}}{\sqrt{E}}+\frac{c_{3}(d, n) M_{2}}{\left(2 \tau_{1} \sqrt{E}\right)^{n-d}} \\
& \text { for } x \in D, \quad 0<\tau_{1} \leq \tau, \quad \sqrt{E} \geq \rho_{1}\left(d, \sigma, M_{1}\right),
\end{aligned}
$$




$$
\begin{aligned}
& \left|v_{1}\left(x, E, \tau_{1}(E)\right)-v(x)\right| \leq\left(c_{2}(d, \sigma) M_{1}^{2}(2 \tau)^{d}+\frac{c_{3}(d, n) M_{2}}{(2 \tau)^{n-d}}\right) E^{-(n-d) /(2 n)} \\
& \text { for } x \in D, \quad \tau_{1}(E)=\tau E^{-(n-1) /(2 n)}, \sqrt{E} \geq \rho_{1}\left(d, \sigma, M_{1}\right)
\end{aligned}
$$

where $0<\tau \leq 1, v_{1}$ is defined by (3.8), $\rho_{1}$ is defined in (2.6), and $c_{2}=c_{2}(d, \sigma), c_{3}=c_{3}(d, n)$ are some positive constants.

Lemma 3.1 is proved in Section 4.

Under the assumptions of Lemma 3.1,

$$
\begin{aligned}
& u_{1}(x, E)=v_{1}\left(x, E, \tau_{1}(E)\right), \quad x \in D, \\
& u_{1}(x, E)=v_{0}(x), \quad x \in \mathbb{R}^{d} \backslash D,
\end{aligned}
$$

can be considered as an optimal reconstruction in the Born approximation for Problem 1.2 with respect to the error decay in $L^{\infty}(D)$ as $E \rightarrow+\infty$.

3.3. Iterative step. The iterative step of our reconstruction is based, in particular, on the following lemma:

Lemma 3.2. Let $v$ satisfy (1.13), $f$ be the scattering amplitude of $v$, and $v_{a p p r}(\cdot, E)$ be an approximation to $v$ such that

$$
\begin{aligned}
& \left|v_{\text {appr }}(x, E)-v(x)\right| \leq \beta E^{-\alpha}, x \in D, \sqrt{E} \geq \rho_{1}(d, \sigma, N), \\
& v_{\text {appr }}(x, E) \equiv v_{0}(x), \quad x \in \mathbb{R}^{d} \backslash D
\end{aligned}
$$

for some $\alpha, \beta>0$ and some $N$ such that

$$
\|v\|_{\sigma} \leq N, \quad\left\|v_{a p p r}(\cdot, E)\right\|_{\sigma} \leq N, \quad \sqrt{E} \geq \rho_{1}(d, \sigma, N)
$$

where $\rho_{1}$ is defined in (2.6). Then the following estimate holds:

$$
\begin{aligned}
& \left|f(k, l)-f_{a p p r}(k, l)+\hat{v}_{a p p r}(k-l, E)-\hat{v}(k-l)\right| \leq \\
& (2 \pi)^{-d} a_{0}(d, \sigma / 2) c_{1}(d, \sigma) c_{4}(D, \sigma) N \beta E^{-\alpha-(1 / 2)}, \\
& (k, l) \in \mathcal{M}_{E}, E^{1 / 2} \geq \rho_{1}(d, \sigma, N),
\end{aligned}
$$

where $f_{\text {appr }}$ is the scattering amplitude for $v_{\text {appr }}, \hat{v}_{\text {appr }}(\cdot, E)$ is the Fourier transform of $v_{\text {appr }}(\cdot, E), \hat{v}$ is the Fourier transform of $v$, (see definition $(1.20)$ ), $c_{4}(D, \sigma)$ is given by (4.12).

Lemma 3.2 is proved in Section 4.

In the iterative step of our reconstruction we assume that $v$ satisfies (1.13), (3.2) and $\|v\|_{\sigma} \leq M_{1},\left\|v-v_{0}\right\|_{n, 1} \leq M_{2}$, as in lemma 3.1 . where

Note that $v_{a p p r}=u_{1}$ of (3.11) satisfies (3.12), (3.13) for $\alpha=\alpha_{1}, \beta=\beta_{1}, N=N_{1}$,

$$
\begin{aligned}
& \alpha_{1}=\frac{n-d}{2 n}, \quad \beta_{1}=c_{2}(d, \sigma) M_{1}^{2}(2 \tau)^{d}+\frac{c_{3}(d, n) M_{2}}{(2 \tau)^{n-d}}, \\
& N_{1}=M_{1}+c_{5}(D, \sigma) \beta_{1}\left(\rho_{1}\left(d, \sigma, M_{1}\right)\right)^{-\alpha_{1}}, \quad c_{5}(D, \sigma)=\sup _{x \in D}\left(1+|x|^{2}\right)^{\sigma / 2} .
\end{aligned}
$$


An iterative approach to non-overdetermined inverse scattering at fixed energy

Then proceeding from the approximation $v_{a p p r}=u_{j}(\cdot, E)$ with number $j$, satisfying (3.12), (3.13) for $\alpha=\alpha_{j}, \beta=\beta_{j}, N=N_{j}$, the approximation $v_{a p p r}=u_{j+1}(\cdot, E)$ with number $j+1$ is constructed as follows:

(1) We find the scattering amplitude $f_{j}$ and the Fourier transform $\hat{u}_{j}(\cdot, E)$ for $u_{j}(\cdot, E)$, where $E^{1 / 2} \geq \rho_{1}\left(d, \sigma, N_{j}\right)$

(2) In a similar way with (3.8), we define

$$
\begin{gathered}
v_{j+1}\left(x, E, \tau_{j+1}\right) \stackrel{\text { def }}{=} v_{j+1}^{+}\left(x, E, \tau_{j+1}\right)+v_{0}^{-}\left(x, 2 \tau_{j+1} \sqrt{E}\right), \\
v_{j+1}^{+}\left(x, E, \tau_{j+1}\right) \stackrel{\text { def }}{=} \int_{p \in \mathbb{R}^{d},|p| \leq 2 \tau_{j+1} \sqrt{E}} e^{-i p x} \times \\
\left(f\left(k_{E}(p), l_{E}(p)\right)-f_{j}\left(k_{E}(p), l_{E}(p)\right)+\hat{u}_{j}\left(k_{E}(p)-l_{E}(p), E\right)\right) d p, \\
x \in D, \quad 0<\tau_{j+1} \leq \tau, \quad E^{1 / 2} \geq \rho_{1}\left(d, \sigma, N_{j}\right),
\end{gathered}
$$

where $v_{0}^{-}, f, k_{E}, l_{E}$ are the same that in (3.8);

(3) Finally, in a similar way with (3.11), we define

$$
\begin{aligned}
& u_{j+1}(x, E)=v_{j+1}\left(x, E, \tau_{j+1}(E)\right), x \in D, \\
& u_{j+1}(x, E)=v_{0}(x), x \in \mathbb{R}^{d} \backslash D, \\
& \tau_{j+1}(E)=\tau E^{-\left(n-1-2 \alpha_{j}\right) /(2 n)},
\end{aligned}
$$

where $E^{1 / 2} \geq \rho_{1}\left(d, \sigma, N_{j}\right)$.

In addition, we have the following lemma:

Lemma 3.3. Under the assumptions of our iterative step, the following estimates hold:

$$
\begin{aligned}
& \qquad\left|v_{j+1}\left(x, E, \tau_{j+1}\right)-v(x)\right| \leq c_{6}(D, \sigma) N_{j} \beta_{j} \frac{\left(2 \tau_{j+1} E^{1 / 2}\right)^{d}}{E^{\alpha_{j}+(1 / 2)}}+\frac{c_{3}(d, n) M_{2}}{\left(2 \tau_{j+1} E^{1 / 2}\right)^{n-d}} \\
& \quad \text { for } x \in D, \quad 0<\tau_{j+1} \leq \tau \leq 1, \quad E^{1 / 2} \geq \rho_{1}\left(d, \sigma, N_{j}\right), \quad j \in \mathbb{N}, \\
& \left|u_{j+1}(x, E)-v(x)\right| \leq\left(c_{6}(D, \sigma) N_{j} \beta_{j}(2 \tau)^{d}+\frac{c_{3}(d, n) M_{2}}{(2 \tau)^{n-d}}\right) E^{-\left(1+2 \alpha_{j}\right)(n-d) /(2 n)} \\
& \text { for } \quad x \in D, \quad E^{1 / 2} \geq \rho_{1}\left(d, \sigma, N_{j}\right), \quad j \in \mathbb{N},
\end{aligned}
$$

where $v_{j+1}, u_{j+1}$ are defined by (3.16), (3.17), $\rho_{1}$ is defined in (2.6).

Lemma 3.3 is proved in Section 5 .

In addition, $u_{j+1}(\cdot, E)$ of (3.17) satisfies (3.12), (3.13) for $\alpha=\alpha_{j+1}, \beta=\beta_{j+1}$, $N=N_{j+1}$, where

$$
\begin{aligned}
& \alpha_{j+1}=\frac{\left(1+2 \alpha_{j}\right)(n-d)}{2 n}, \quad \beta_{j+1}=c_{6}(D, \sigma) N_{j} \beta_{j}(2 \tau)^{d}+\frac{c_{3}(d, n) M_{2}}{(2 \tau)^{n-d}}, \\
& N_{j+1}=M_{1}+c_{5}(D, \sigma) \max _{1 \leq i \leq j} \beta_{i}\left(\rho_{1}\left(d, \sigma, N_{i}\right)\right)^{-\alpha_{i}}, \quad j \in \mathbb{N} .
\end{aligned}
$$


Note also that

$$
N_{j_{1}} \leq N_{j_{2}}, \quad \rho_{1}\left(d, \sigma, N_{j_{1}}\right) \leq \rho_{1}\left(d, \sigma, N_{j_{2}}\right) \text { for } 1 \leq j_{1} \leq j_{2}
$$

3.4. Final theorem. Proceeding from lemmas 3.1, 3.2, 3.3 and formulas (3.15), (3.20), (3.21) we obtain the following theorem:

Theorem 3.1. Let $v$ satisfy (1.13), (3.1) and $\|v\|_{\sigma} \leq M_{1},\left\|v-v_{0}\right\|_{n, 1} \leq M_{2}$. Let $u_{j}(\cdot, E)$ be constructed from $\left.f\right|_{\Gamma_{E}^{\delta(E)}}$ by the iterations of subsections 3.2, 3.3, where $f$ is the scattering amplitude for $v, \delta(E)=\tau E^{-(d-1) /(2 d)}, 0<\tau \leq 1$, and $j \in \mathbb{N}$; see formulas (3.11), (3.16), (3.17). Then the following estimates hold:

$$
\left\|u_{j}(\cdot, E)-v\right\|_{L^{\infty}(D)} \leq \beta_{j} E^{-\alpha_{j}} \text { for } E^{1 / 2} \geq \rho_{1}\left(d, \sigma, N_{j-1}\right)
$$

where

$$
\alpha_{j}=\left(1-\left(\frac{n-d}{n}\right)^{j}\right) \frac{n-d}{2 d},
$$

$\beta_{j}=\beta_{j}\left(M_{1}, M_{2}, D, \sigma, n, \tau\right), N_{j}=N_{j}\left(M_{1}, M_{2}, D, \sigma, n, \tau\right)$ are constructed recurrently via (3.15), (3.20) (with $N_{0}=M_{1}$ ).

Theorem 3.1 is proved in Section 5 .

3.5. Explicit formulas. One can see that:

(1) $u_{1}(\cdot, E)$ is constructed by explicit formulas from $\left.f\right|_{\Gamma_{E}^{\delta(E)}}$ and $v_{0}$ for $\sqrt{E} \geq \rho_{1}\left(d, \sigma, M_{1}\right)$ (see Subsection 3.2 and formula (5.9)) and

(2) $u_{j+1}(\cdot, E)$ is constructed by explicit formulas from $u_{j}(\cdot, E),\left.f\right|_{\Gamma_{E}^{\delta(E)}},\left.f_{j}\right|_{\Gamma_{E}^{\delta(E)}}$ and $v_{0}$ for $\sqrt{E} \geq \rho_{1}\left(d, \sigma, N_{j}\right)$ and each $j \in \mathbb{N}$ (see Subsection 3.3 and formula (5.9)). However, $f_{j}$ is constructed from $u_{j}$ via $(1.5)$, (1.6) with $\psi^{+}=\psi_{j}^{+}, v=v_{j}$, where (1.5) is not yet an explicit formula for $\psi_{j}^{+}$.

Thus the construction of $u_{j}(\cdot, E), j \geq 2$, of Subsections 3.3, 3.4 is not reduced yet to explicit formulas. In order to have a similar construction involving explicit formulas only we can proceed as follows. Instead of $u_{j}, j \geq 2$, we can construct $\tilde{u}_{j}(\cdot, E), j \geq 2$, $\sqrt{E} \geq \rho_{1}\left(d, \sigma, M_{1}\right)$, via $(3.16),(3.17)$ with $f_{j}, \hat{u}_{j}, u_{j+1}$ replaced by $\tilde{f}_{j}^{a p p r}, \hat{\tilde{u}}_{j}, \tilde{u}_{j+1}$, where $\hat{\tilde{u}}_{j}$ is the Fourier transform of $\tilde{u}_{j}$ as before, whereas $\tilde{f}_{j}^{a p p r}$ is the approximation to the scattering amplitude $\tilde{f}_{j}$ of $\tilde{u}_{j}$, defined as follows. Proceeding from (1.6), (1.5), (2.2), (2.5), we define

$$
\begin{aligned}
& \tilde{f}_{j}^{a p p r}(k, l)=(2 \pi)^{-d} \int_{\mathbb{R}^{d}} e^{-i l y} \Lambda^{\sigma / 2} v(y) \tilde{\varphi}_{j}^{a p p r}(y, k) d y, \\
& \tilde{\varphi}_{j}^{a p p r}(\cdot, k)=\sum_{\nu=0}^{m_{j}}\left(\tilde{A}_{j}(k)\right)^{\nu} e(\cdot, k),
\end{aligned}
$$


where $(k, l) \in \mathcal{M}_{E}, m_{j}$ is the minimal natural number such that $m_{j} \geq 2 \alpha_{j}, \tilde{A}_{j}(k)$ is defined as $A(k)$ of $(2.2)$ with $v$ replaced by $\tilde{u}_{j}, e(\cdot, k)$ is defined in $(2.2)$. Here $\alpha_{j}$ is the number of $(3.23), j \in \mathbb{N}$.

Using estimate $(2.5)$ with $v$ replaced by $\tilde{u}_{j}$ and using the proof of Lemma 3.3, one can show that

$$
\left\|\tilde{u}_{j}(\cdot, E)-v\right\|_{L^{\infty}(D)}=O\left(E^{-\alpha_{j}}\right) \text { as } E \rightarrow+\infty,
$$

as for the initial $u_{j}$. However, now because of the finite sum in $(3.25), \tilde{u}_{j}$ is constructed via a finite number of explicite formulas for each $j \geq 2$.

\section{Proofs of Lemmas 3.1 and 3.2}

Proof of lemma 3.1. Due to (3.6), we have that

$$
v(x)=v^{+}\left(x, 2 \tau_{1} \sqrt{E}\right)+v_{0}^{-}\left(x, 2 \tau_{1} \sqrt{E}\right)+w^{-}\left(x, 2 \tau_{1} \sqrt{E}\right), \quad x \in D .
$$

Due to (3.8), (4.1), (3.5), we have that

$$
\begin{aligned}
& v_{1}\left(x, E, \tau_{1}\right)-v(x)=\delta_{1}^{+} v\left(x, E, \tau_{1}\right)+\delta_{1}^{-} v\left(x, E, \tau_{1}\right), \\
& \delta_{1}^{+} v\left(x, E, \tau_{1}\right) \stackrel{\text { def }}{=} \int_{p \in \mathbb{R}^{d},|p| \leq 2 \tau_{1} \sqrt{E}} e^{-i p x}\left(f\left(k_{E}(p), l_{E}(p)\right)-\hat{v}(p)\right) d p, \\
& \delta_{1}^{-} v\left(x, E, \tau_{1}\right) \stackrel{\text { def }}{=}-w^{-}\left(x, 2 \tau_{1} \sqrt{E}\right), \quad x \in D .
\end{aligned}
$$

Using (2.11) and the definitions of $k_{E}(p), l_{E}(p)$ of (1.8) we obtain that

$$
\begin{aligned}
& \left|\delta_{1}^{+}\left(x, E, \tau_{1}\right)\right| \leq 2(2 \pi)^{-d} a_{0}(d, \sigma / 2)\left(c_{1}(d, \sigma)\|v\|_{\sigma}\right) E^{-1 / 2} \times \\
& \left(2 \tau_{1} \sqrt{E}\right)^{d}\left|\mathcal{B}_{1}\right|, \quad x \in D, \quad \sqrt{E} \geq \rho_{1}\left(d, \sigma,\|v\|_{\sigma}\right)
\end{aligned}
$$

where $\left|\mathcal{B}_{1}\right|$ denotes the standard Euclidean volume of $\mathcal{B}_{1}$, i.e.

$$
\left|\mathcal{B}_{1}\right|=\int_{p \in \mathbb{R}^{d},|p| \leq 1} d p .
$$

In order to estimate $\delta_{1}^{-} v\left(x, E, \tau_{1}\right)$ we use that if $w \in W^{n, 1}\left(\mathbb{R}^{d}\right)$, then

$$
|\hat{w}(p)| \leq a_{1}(n, d)\|w\|_{n, 1}(1+|p|)^{-n}, \quad p \in \mathbb{R}^{d}
$$

Using the definition of $w^{-}$of (3.3)-(3.5) and estimate (4.5) we obtain that

$$
\begin{aligned}
& \left|\delta_{1}^{-}\left(x, E, \tau_{1}\right)\right| \leq \int_{p \in \mathbb{R}^{d},|p| \geq 2 \tau_{1} \sqrt{E}} \frac{a_{1}(n, d)\left\|v-v_{0}\right\|_{n, 1}}{(1+|p|)^{n}} d p \leq \\
& \left|\mathbb{S}^{d-1}\right| \frac{a_{1}(n, d)\left\|v-v_{0}\right\|_{n, 1}}{n-d} \frac{1}{\left(2 \tau_{1} \sqrt{E}\right)^{n-d}}, \quad x \in D,
\end{aligned}
$$


where $\left|\mathbb{S}^{d-1}\right|$ denotes the standard Euclidean volume of $\mathbb{S}^{d-1}$, i.e.

$$
\left|\mathbb{S}^{d-1}\right|=\int_{\theta \in \mathbb{S}^{d-1}} d \theta .
$$

Estimate (3.9) follows from (4.2), (4.3), (4.6). Estimate (3.10) follows from (3.9).

Lemma 3.1 is proved.

Proof of Lemma 3.2. Using formula (1.6) for the scattering amplitude we obtain that

$$
\begin{aligned}
& f(k, l)=\hat{v}(k-l)+\delta f(k, l), \\
& \delta f(k, l) \stackrel{\text { def }}{=}(2 \pi)^{-d} \int_{\mathbb{R}^{d}} e^{-i l x} v(x)\left(\psi^{+}(x, k)-e^{i k x}\right) d x, \\
& f_{\text {appr }}(k, l)=\hat{v}(k-l, E)+\delta f_{\text {appr }}(k, l), \\
& \delta f_{\text {appr }}(k, l) \stackrel{\text { def }}{=}(2 \pi)^{-d} \int_{\mathbb{R}^{d}} e^{-i l x} v_{a p p r}(x, E)\left(\psi_{a p p r}^{+}(x, k)-e^{i k x}\right) d x, \\
& (k, l) \in \mathcal{M}_{E}, \quad \sqrt{E} \geq \rho_{1}(d, \sigma, N),
\end{aligned}
$$

where $\psi_{a p p r}^{+}$denotes the scattering solutions for $v_{a p p r}$.

In addition, we have that

$$
\begin{gathered}
\delta f(k, l)-\delta f_{\text {appr }}(k, l)= \\
(2 \pi)^{-d} \int_{\mathbb{R}^{d}} e^{-i l x}\left(v(x)-v_{a p p r}(x, E)\right)\left(\psi^{+}(x, k)-e^{i k x}\right) d x+ \\
(2 \pi)^{-d} \int_{\mathbb{R}^{d}} e^{-i l x} v_{\text {appr }}(x, E)\left(\psi^{+}(x, k)-\psi_{\text {appr }}^{+}(x, k)\right) d x, \\
\left|\delta f(k, l)-\delta f_{a p p r}(k, l)\right| \leq \\
(2 \pi)^{-d} \int_{\mathbb{R}^{d}} \Lambda^{\sigma / 2}\left|v(x)-v_{a p p r}(x, E)\right| \Lambda^{-\sigma / 2}\left|\psi^{+}(x, k)-e^{i k x}\right| d x+ \\
(2 \pi)^{-d} \int_{\mathbb{R}^{d}} \Lambda^{\sigma / 2}\left|v_{a p p r}(x, E)\right| \Lambda^{-\sigma / 2}\left|\psi^{+}(x, k)-\psi_{a p p r}^{+}(x, k)\right| d x,
\end{gathered}
$$

where $\Lambda$ denotes the function $\left(1+|x|^{2}\right)^{1 / 2}$. Using (4.10), (2.5) for $m=0,(2.6),(2.9)$ for $v_{1}=v, v_{2}=v_{a p p r},(3.12),(3.13)$, we obtain that

$$
\begin{aligned}
& \left|\delta f(k, l)-\delta f_{\text {appr }}(k, l)\right| \leq \\
& 2(2 \pi)^{-d} a_{0}(d, \sigma / 2) c_{1}(d, \sigma)\left\|\Lambda^{\sigma / 2}\right\|_{L^{2}(D)} N \beta E^{-\alpha-(1 / 2)}+ \\
& 4(2 \pi)^{-d} a_{0}(d, \sigma / 2) c_{1}(d, \sigma)\left\|\Lambda^{-\sigma / 2}\right\|_{L^{2}\left(\mathbb{R}^{d}\right)}\left\|\Lambda^{\sigma}\right\|_{L^{\infty}(D)} N \beta E^{-\alpha-(1 / 2)}= \\
& (2 \pi)^{-d} a_{0}(d, \sigma / 2) c_{1}(d, \sigma) c_{4}(D, \sigma) N \beta E^{-\alpha-(1 / 2)}
\end{aligned}
$$


An iterative approach to non-overdetermined inverse scattering at fixed energy

for $(k, l) \in \mathcal{M}_{E}, \sqrt{E} \geq \rho_{1}(d, \sigma, N)$, where

$$
c_{4}(D, \sigma)=2\left\|\Lambda^{\sigma / 2}\right\|_{L^{2}(D)}+4\left\|\Lambda^{-\sigma / 2}\right\|_{L^{2}\left(\mathbb{R}^{d}\right)}\left\|\Lambda^{\sigma}\right\|_{L^{\infty}(D)} .
$$

Note also that

$$
\left\|\Lambda^{-\sigma / 2}\right\|_{L^{2}\left(\mathbb{R}^{d}\right)}=c_{1}(d, \sigma), \quad\left\|\Lambda^{\sigma / 2}\right\|_{L^{2}(D)} \leq\left\|\Lambda^{\sigma}\right\|_{L^{\infty}(D)}\left\|\Lambda^{-\sigma / 2}\right\|_{L^{2}\left(\mathbb{R}^{d}\right)} .
$$

Estimate (3.14) follows from (4.8), (4.11).

Lemma 3.2 is proved.

\section{Proofs of Lemma 3.3 and Theorem 3.1}

Proof of Lemma 3.3. In a completely similar way with (4.1) we have that

$$
v(x)=v^{+}\left(x, 2 \tau_{j+1} \sqrt{E}\right)+v_{0}^{-}\left(x, 2 \tau_{j+1} \sqrt{E}\right)+w^{-}\left(x, 2 \tau_{j+1} \sqrt{E}\right), \quad x \in D,
$$

where $v^{+}, v_{0}^{-}, w^{-}$are the functions of (3.4), (3.6). Due to (3.16), (5.1), (3.5), in a similar way with (4.2) we have that

$$
\begin{aligned}
& v_{j+1}\left(x, E, \tau_{j+1}\right)-v(x)=\delta_{j+1}^{+} v\left(x, E, \tau_{j+1}\right)+\delta_{j+1}^{-} v\left(x, E, \tau_{j+1}\right), \\
& \delta_{j+1}^{+} v\left(x, E, \tau_{j+1}\right) \stackrel{\text { def }}{=} \int_{p \in \mathbb{R}^{d},|p| \leq 2 \tau_{j+1} \sqrt{E}} e^{-i p x} \times \\
& \left(f\left(k_{E}(p), l_{E}(p)\right)-f_{j}\left(k_{E}(p), l_{E}(p)\right)+\hat{u}_{j}\left(k_{E}(p)-l_{E}(p), E\right)-\hat{v}(p)\right) d p, \\
& \delta_{j+1}^{-} v\left(x, E, \tau_{j+1}\right) \stackrel{\text { def }}{=}-w^{-}\left(x, 2 \tau_{j+1} \sqrt{E}\right), \quad x \in D .
\end{aligned}
$$

Using (3.14) for $f_{\text {appr }}=f_{j}, \hat{v}_{\text {appr }}=\hat{u}_{j}, \alpha=\alpha_{j}, \beta=\beta_{j}, N=N_{j}$ and using the definitions of $k_{E}(p), l_{E}(p)$ of (1.8), in a similar way with (4.3) we have that

$$
\begin{aligned}
& \left|\delta_{j+1}^{+} v\left(x, E, \tau_{j+1}\right)\right| \leq c_{6}(D, \sigma) N_{j} \beta_{j} E^{-\alpha_{j}-(1 / 2)}\left(2 \tau_{j+1} \sqrt{E}\right)^{d} \\
& x \in D, \quad \sqrt{E} \geq \rho_{1}\left(d, \sigma, N_{j}\right)
\end{aligned}
$$

where

$$
c_{6}(D, \sigma)=(2 \pi)^{-d} a_{0}(d, \sigma / 2) c_{1}(d, \sigma) c_{4}(D, \sigma)\left|\mathcal{B}_{1}\right| .
$$

In addition, in a completely similar way with (4.6) we have that

$$
\left|\delta_{j+1}^{-} v\left(x, E, \tau_{j+1}\right)\right| \leq\left|\mathbb{S}^{d-1}\right| \frac{a_{1}(n, d)\left\|v-v_{0}\right\|_{n, 1}}{n-d} \frac{1}{\left(2 \tau_{j+1} \sqrt{E}\right)^{n-d}}, \quad x \in D .
$$

Estimate (3.18) follows from (5.3), (5.5). Estimate (3.19) follows from (3.18), (3.17) and from the identities

$$
\begin{aligned}
& E^{-\left(n-1-2 \alpha_{j}\right) /(2 n)} E^{1 / 2}=E^{\left(1+2 \alpha_{j}\right) /(2 n)}, \\
& E^{d\left(1+2 \alpha_{j}\right) /(2 n)} E^{-\alpha_{j}-(1 / 2)}=E^{-\left(1+2 \alpha_{j}\right)(n-d) /(2 n)} .
\end{aligned}
$$


This completes the proof of Lemma 3.3.

Proof of Theorem 3.1. Due to (3.15), (3.20), we have that

$$
\alpha_{1}=\frac{n-d}{2 n}, \quad \alpha_{j+1}=\frac{n-d}{2 n}+\alpha_{j} \frac{n-d}{n}, \quad j \in \mathbb{N} .
$$

Formulas (5.7) imply (3.23). Indeed, the sequence $\alpha_{j}, j \in \mathbb{N}$, is uniquely defined by (5.7) and $\alpha_{j}$ of (3.23) satisfy (5.7). In particular,

$$
\begin{aligned}
& \frac{n-d}{2 n}+\left(1-\left(\frac{n-d}{n}\right)^{j}\right) \frac{n-d}{2 d} \frac{n-d}{n}= \\
& \frac{n-d}{2 n}+\frac{n-d}{2 d} \frac{n-d}{n}-\left(\frac{n-d}{n}\right)^{j+1} \frac{n-d}{2 d}= \\
& \left(1-\left(\frac{n-d}{n}\right)^{j+1}\right) \frac{n-d}{2 d}, \quad j \in \mathbb{N} .
\end{aligned}
$$

Next, due to $(5.7),(3.23)$ and due to the definition of $\tau_{j}(E), j \in \mathbb{N}$, of $(3.10),(3.17)$ we have that

$$
\tau_{j_{1}}(E)=\tau E^{-\left(n-1-2 \alpha_{j_{1}}\right) /(2 n)} \leq \tau_{j_{2}}(E)=\tau E^{-\left(n-1-2 \alpha_{j_{2}}\right) /(2 n)} \leq \delta(E)=\tau E^{-(d-1) /(2 d)}
$$

for $0<\tau \leq 1,1 \leq j_{1} \leq j_{2}, E \geq 1$.

Using the definition of $u_{j}, j \in \mathbb{N}$, of (3.11), (3.17) and using inequalities of (3.21) and (5.9) we obtain that $u_{j}(\cdot, E)$ are correctly defined in terms of $\left.f\right|_{\Gamma_{E}^{\delta(E)}}$ for $E^{1 / 2} \geq \rho_{1}\left(d, \sigma, N_{j-1}\right), j \in \mathbb{N}$. $(3.20)$.

Finally, estimates (3.22) follow from estimates (3.10), (3.19) and formulas (3.15),

This completes the proof of Theorem 3.1.

\section{Acknowledgements}

This work was partially supported by TFP No 14.A18.21.0866 of Ministry of Education and Sciences of Russian Federation (at Faculty of Control and Applied Mathematics of Moscow Institute of Physics and Technology).

\section{References}

[ABR] N.V. Alexeenko, V.A. Burov, O.D. Rumyantseva, Solution of the three-dimensional acoustical inverse scattering problem. The modified Novikov algorithm, Acoust. J. 54(3), 2008, 469-482 (in Russian), English transl.: Acoust. Phys. 54(3), 2008, 407-419.

[BK] L. Beilina, M.V. Klibanov, Approximate global convergence and adaptivity for coefficient inverse problems, Springer (New York), 2012.

[BS] F.A. Berezin, M.A. Shubin, The Schrödinger Equation, Vol. 66 of Mathematics and Its Applications, Kluwer Academic, Dordrecht, 1991. 
[B] A.L. Buckhgeim, Recovering a potential from Cauchy data in the two-dimensional case, J. Inverse Ill-Posed Probl. 16(1), 2008, 19-33.

[BAR] V.A. Burov, N.V. Alekseenko, O.D. Rumyantseva, Multifrequency generalization of the Novikov algorithm for the two-dimensional inverse scattering problem, Acoustical Physics 55(6), 2009, 843-856.

[ChS] K. Chadan, P.C. Sabatier, Inverse Problems in Quantum Scattering Theory, 2nd edn. Springer, Berlin, 1989.

[DKN] B.A. Dubrovin, I.M. Krichever, S.P. Novikov, The Schrödinger equation in a periodic field and Riemann surface, Dokl. Akad. Nauk SSSR 229, 1976, 15-18 (in Russian), English transl.: Sov. Math. Dokl. 17, 1976, 947-951.

[ E] G. Eskin, Lectures on Linear Partial Differential Equations, Graduate Studies in Mathematics, Vol.123, American Mathematical Society, 2011.

[ER1] G. Eskin, J. Ralston, Inverse backscattering problem in three dimensions, Commun. Math. Phys. 124, 1989, 169-215.

[ER2] G. Eskin, J. Ralston, Inverse scattering problem for the Schrödinger equation with magnetic potential at a fixed energy, Commun. Math. Phys. 173, 1995, 199-224.

[F1] L.D. Faddeev, Uniqueness of the solution of the inverse scattering problem, Vest. Leningrad Univ. 7, 1956, 126-130 [in Russian].

[F2] L.D. Faddeev, The inverse problem in the quantum theory of scattering.II, Current problems in mathematics, Vol. 3, 1974, pp. 93-180, 259. Akad. Nauk SSSR Vsesojuz. Inst. Naucn. i Tehn. Informacii, Moscow(in Russian); English Transl.: J.Sov. Math. 5, 1976, 334-396.

[FM] L.D. Faddeev, S.P. Merkuriev, Quantum Scattering Theory for Multi-particle Systems", Nauka, Moscow, 1985 [in Russian].

[GHN] A.A. Gonchar, N.N. Novikova, G.M. Henkin, Multipoint Padé approximations in the inverse Sturm-Liouville problem, Mat. Sb. 182(8), 1991, 1118-1128 (in Russian); English transl.: Mathematics of the USSR - Sbornik 73(2), 1992, 479-489.

[ G] P.G. Grinevich, The scattering transform for the two-dimensional Schrödinger operator with a potential that decreases at infinity at fixed nonzero energy, Uspekhi Mat. Nauk 55:6(336),2000, 3-70 (Russian); English translation: Russian Math. Surveys 55:6, 2000, 1015-1083.

[ HH] P. Hähner, T. Hohage, New stability estimates for the inverse acoustic inhomogeneous medium problem and applications, SIAM J. Math. Anal., 33(3), 2001, 670-685.

[ HN] G.M. Henkin, R.G. Novikov, The $\bar{\partial}$-equation in the multidimensional inverse scattering problem, Uspekhi Mat. Nauk 42(3), 1987, 93-152 (in Russian); English Transl.: Russ. Math. Surv. 42(3), 1987, 109-180.

[ I] M.I. Isaev, Exponential instability in the inverse scattering problem on the energy interval, Func. Analiz i ego Pril.(to appear), arXiv:1012.5526.

[ IN] M.I. Isaev, R.G. Novikov New global stability estimates for monochromatic inverse acoustic scattering, SIAM J. Math. Anal. 45(3), 2013, 1495-1504.

[J] A. Jensen, High energy resolvent estimates for generalized many-body Schrödinger operators, Publ. RIMS Kyoto Univ. 25, 1989, 155-167.

[K] M.V. Klibanov, Carleman estimates for global uniqueness, stability and numerical methods for coefficient inverse problems, J. Inverse and Ill-posed Probl., (to appear). 
[M] H.E. Moses, Calculation of the scattering potential from reflection coefficients, Phys. Rev. 102, 1956, 559-567.

[LRS] M.M. Lavrentev, V.G. Romanov, S.P. Shishatskii, Ill-posed problems of mathematical physics and analysis, Translated from the Russian by J.R. Schulenberger. Translation edited by Levi J. Leifman. Translations of Mathematical Monographs, 64. American Mathematical Society, Providence, RI, 1986.

[ N1] R.G. Novikov, Multidimensional inverse spectral problem for the equation $-\Delta \psi+$ $(v(x)-E u(x)) \psi=0$, Funkt. Anal. Prilozhen. 22(4), 1988, 11-22 (in Russian); Engl. Transl. Funct. Anal. Appl. 22, 1988, 263-272.

[ N2] R.G. Novikov, The inverse scattering problem at fixed energy level for the twodimensional Schrödinger operator, J. Funct. Anal., 103, 1992, 409-463.

[ N3] R.G. Novikov, The inverse scattering problem at fixed energy for Schrödinger equation with an exponentially decreasing potential, Comm. Math. Phys., 161, 1994, 569-595.

[ N4] R.G. Novikov, Rapidly converging approximation in inverse quantum scattering in dimension 2. Physics Letters A 238, 1998, 73-78.

[ N5] R.G. Novikov, The $\bar{\partial}$-approach to approximate inverse scattering at fixed energy in three dimensions, IMRP Int. Math. Res. Pap. 2005, no. 6, 287-349.

[ N6] R.G. Novikov, On non-overdetermined inverse scattering at zero energy in three dimensions, Ann. Scuola Norm. Sup. Pisa CL. Sci. 5, 2006, 279-328.

[ N7] R.G. Novikov, On iterative reconstruction in the nonlinearized polarization tomography, Inverse Problems 25, 2009, 115010 (18pp).

[ N8] R.G. Novikov, Approximate Lipschitz stability for non-overdetermined inverse scattering at fixed energy, J. Inverse Ill-Posed Probl., DOI 10.1515/jip-2012-0101.

[ NS] R.G. Novikov, M. Santacesaria, Monochromatic reconstruction algorithms for twodimensional multi-channel inverse problems, Int. Math. Res. Notices 2013(6), 2013, 1205-1229.

[ R] T. Regge, Introduction to complex orbital moments, Nuovo Cimento 14, 1959, 951976.

[S1] P. Stefanov, Stability of the inverse problem in potential scattering at fixed energy, Annales de l'Institut Fourier, tome 40(4), 1990, 867-884.

[ S2] P. Stefanov, A uniqueness result for the inverse back-scattering problem, Inverse Problems 6, 1990, 1055-1064.

[ VW] A.Vasy, X.-P. Wang, Inverse scattering with fixed energy for dilation-analytic potentials, Inverse Problems, 20, 2004, 1349-1354.

[W] R. Weder, Global uniqueness at fixed energy in multidimensional inverse scattering theory, Inverse Problems 7, 1991, 927-938.

[ WY] R. Weder, D. Yafaev, On inverse scattering at a fixed energy for potentials with a regular behaviour at infinity, Inverse Problems, 21, 2005, 1937-1952. 